Supporting Information for

\title{
Electrons generate self-complementary broadband vortex light beams using chiral photon sieves
}

\author{
Nika van Nielen ${ }^{1}$, Mario Hentschel $^{2}$, Nick Schilder ${ }^{2}$, Harald Giessen $^{2}$, Albert Polman ${ }^{1}$, Nahid \\ Talebi $^{3 *}$ \\ ${ }^{1}$ Center for Nanophotonics, AMOLF, Science Park 104, 1098 XG Amsterdam, The Netherlands \\ ${ }^{2} 4^{\text {th }}$ Physics Institute and Research Center SCoPE, University of Stuttgart, Pfaffenwaldring 57, 70550 \\ Stuttgart, Germany \\ ${ }^{3}$ Institute of Experimental and Applied Physics, Christian Albrechts University, Leibnizstr. 19, 24118 Kiel, \\ Germany \\ E-mail: talebi@physik.uni-kiel.de
}

\section{3D field distributions of electron-generated vortex beam}

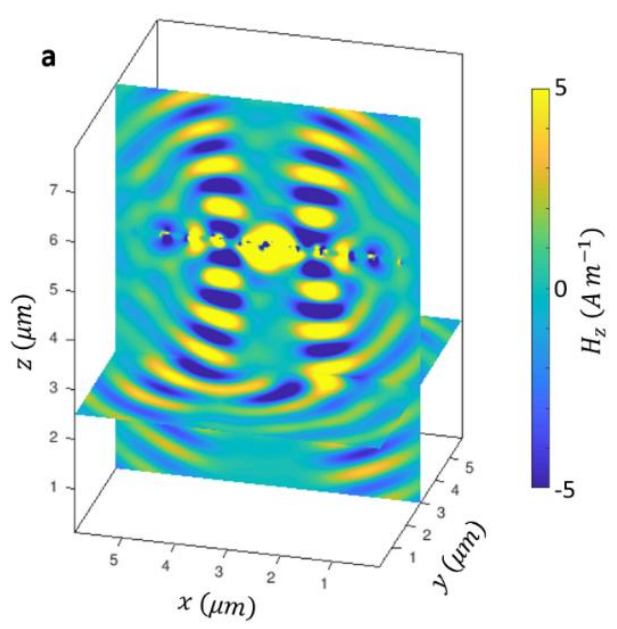

Figure S1. 3D spatial distribution of the z-component of the magnetic field $\left(H_{z}\right)$ in $x y$ - and $y z$ - planes for a right-handed spiral with $l=4$, demonstrating the directionality of the $\mathrm{TE}_{\mathrm{z}}$ waves.

\section{Interference of Laguerre-Gauss beams with transition radiation}

Normally, optical waves with topological charges in free space are specified with LG beams, with the distribution described with Eq. (1) in the main text. Fig. S2 a, b shows the intensity and phase of a LG beam with the topological charge of $l=4$, plotted in the perspective of the origin looking in the direction of propagation. However, in the experimental and calculated CL patterns, we directly observe a spiral pattern with the number of arms following exactly the value for $l$, plotted in the opposite perspective of the detector looking towards the source. We show here that this is due to the interference between the 
generated LG waves and transition radiation, resulting in the encoding of the phase of the LG waves into the detected intensity profile (Figure S2 c). We have represented the transition radiation with an expanded spherical beam and the total field intensity has been derived as a coherent superposition of the spherical beam and the LG wave.
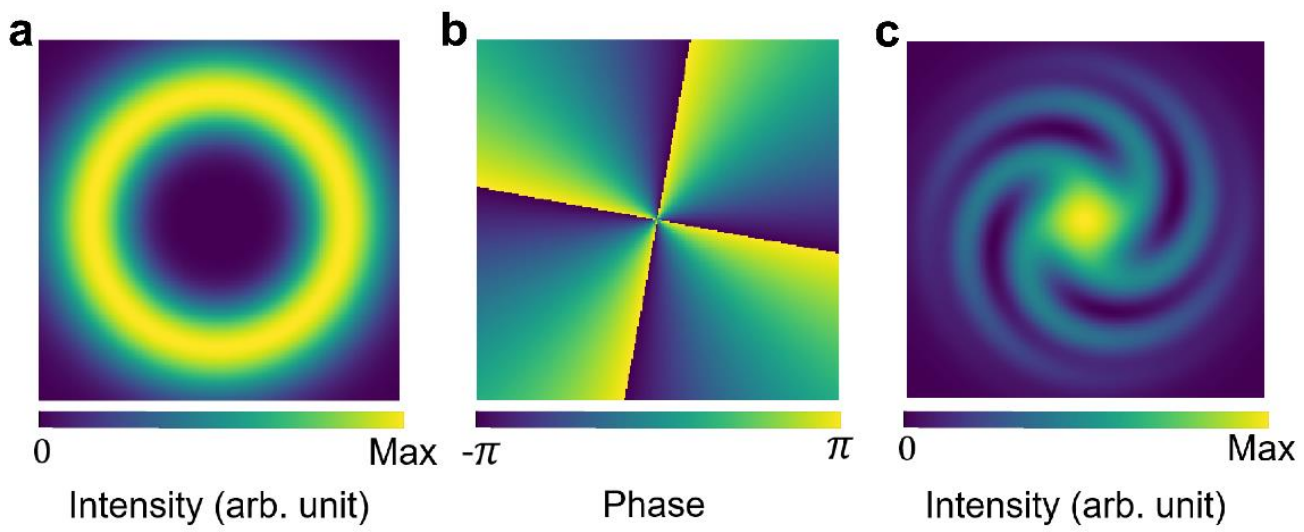

Figure S2. Interference of LG waves with transition radiations leads to the appearance of characteristic winding arms in the intensity profile. (a) Intensity of LG wave with $l=4$; (b) Phase of LG wave with $l=4$; (c) Intensity of interfering LG beam and expanded spherical beam.

\section{Surface plasmon polariton propagation constant for vacuum/Au/Si ${ }_{3} \mathrm{~N}_{4} /$ vacuum layer stack.}

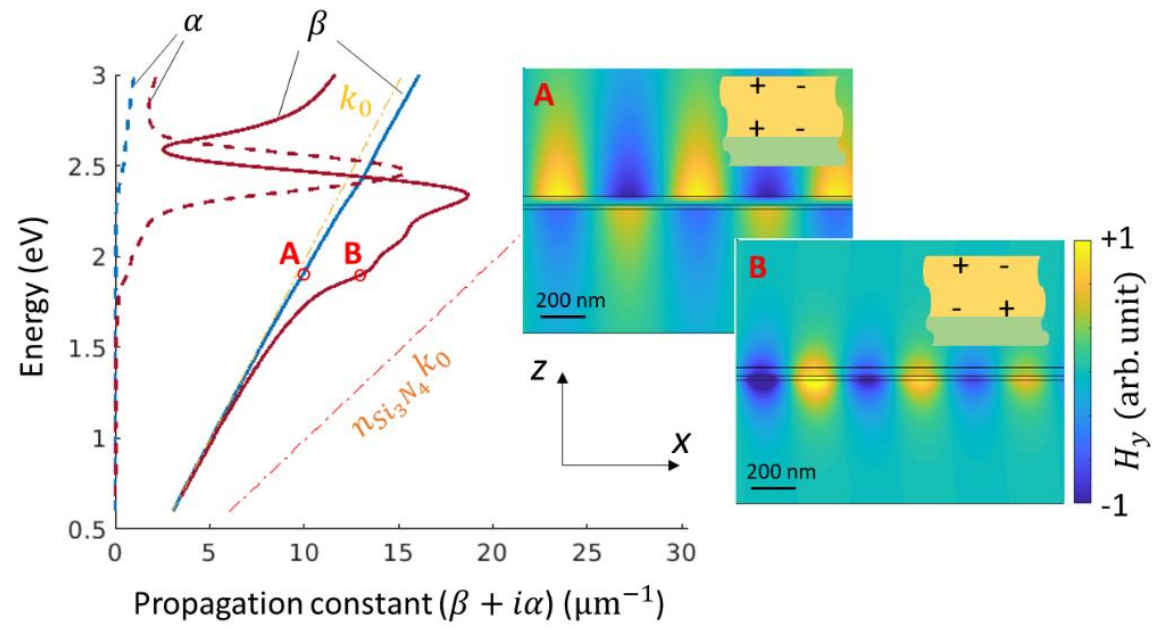

Fig. S3. Numerically calculated propagation constant (real part $\beta$, imaginary part $\alpha$ ) of SPPs with symmetric (blue) and anti-symmetric (red) charge distributions supported by the vacuum/Au/Si3 $\mathrm{N}_{4} /$ vacuum structure. The light dispersion in vacuum (yellow dashed line) and in $\mathrm{Si}_{3} \mathrm{~N}_{4}$ (orange dashed line) are also indicated. The attenuation constant of the quasi-symmetric mode is much smaller than that of the quasi-antisymmetric mode. The inset shows calculated time snapshots of the magnetic field $\mathrm{H}_{\mathrm{y}}$ for the symmetric (A) and anti-symmetric (B) modes. 


\section{Methods}

Sample Fabrication First, a $40 \mathrm{~nm}$ thick gold film is deposited via electron beam evaporation onto a 20 $\mathrm{nm}$ thick silicon nitride $\left(\mathrm{Si}_{3} \mathrm{~N}_{4}\right)$ TEM membrane. Due to the fragility of the membrane, structuring is performed by direct milling with a focused ion beam. In order to achieve the required high resolution we utilized the Raith ionLine Plus ion beam structuring system. The holes where milled using a focused beam of $\mathrm{Au}^{++}$ions at an acceleration voltage of $35 \mathrm{kV}$ and a beam-limiting aperture size of 7 micrometers. For some of the structures, the spiral pattern is surrounded by a circular trench which is milled all the way through the gold film but only partially into the silicon nitride membrane.

Cathodoluminescence The CL setup is based on a Thermo Fisher Quanta650 SEM with a Schottky field emission gun electron source, equipped with a Delmic SPARC optical detection system. The electron energy is $30 \mathrm{keV}$. The primary light collection inside the microscope is performed by an off-axis aluminum paraboloid mirror that is mounted on a micromanipulation system. The mirror has an acceptance angle of $1.46 \pi \mathrm{sr}$, a focal distance of $0.5 \mathrm{~mm}$, and a $600 \mu \mathrm{m}$ diameter hole above the focal point for supporting the electron excitation. A specially designed sample holder was used for all but two of the $\mathrm{CL}$ maps $(l=$ 2 and $l=4$ maps at $\lambda=650 \mathrm{~nm}$ ) to ensure that backreflection of CL from the holder was minimized. For angle-resolved $\mathrm{CL}$ measurements the optical beam is passed through color filters to select a specific wavelength (bandwidth $40 \mathrm{~nm}$ ), and directed onto a 2D back-illuminated, Peltier-cooled, silicon CCD array detector ${ }^{1}$.

Simulations For simulating the electron-induced fields, we have used a semi-classical representation ${ }^{2}$ where the electron is modeled by a current density distribution associated with a moving charge at a kinetic energy of $E=30 \mathrm{keV}$, similar to the experiment. We assumed transverse and longitudinal broadenings of $2 \mathrm{~nm}$ or $20 \mathrm{~nm}$, respectively. We performed FDTD simulations using a home-built code to gain an insight into the temporal distribution of the generated electron-induced radiation. The mesh size is $2 \mathrm{~nm}$. The simulation domain was terminated by using higher-order absorbing boundary conditions. The dielectric function of gold was modeled by a Drude function in addition to two critical point functions ${ }^{3,4}$. For calculating angular CL distributions, the field distributions at a plane normal to the electron trajectory are calculated, and are then transformed into the frequency domain using discrete Fourier transformation. To achieve convergence, 10,000 time iterations are employed.

\section{References:}

(1) Coenen, T.; Vesseur, E. J. R.; Polman, A. Angle-resolved cathodoluminescence spectroscopy. Appl. Phys. Lett. 2011, 99 (14), 143103.

(2) Talebi, N.; Sigle, W.; Vogelgesang, R.; van Aken, P. Numerical simulations of interference effects in photon-assisted electron energy-loss spectroscopy. New J. Phys. 2013, 15 (5), 053013.

(3) Etchegoin, P. G.; Ru, E. C. L.; Meyer, M. An analytic model for the optical properties of gold. J. Chem. Phys. 2006, 125 (16), 164705.

(4) Etchegoin, P. G.; Ru, E. C. L.; Meyer, M. Erratum: "An analytic model for the optical properties of gold” [J. Chem. Phys. 125, 164705 (2006)]. J. Chem. Phys. 2007, 127 (18), 189901. 